Results Of the 2647 unique studies screened for inclusion, 266 full-texts were assessed for eligibility and 33 studies were included in qualitative data analysis. Among 28 studies of reproductive-aged women using $\mathrm{HCs}, 15 / 28$ reported on $>1$ $\mathrm{HC}$ type. Oestrogen-containing contraceptives, mostly reflecting the combined-oral contraceptive pill, had a positive effect on the VM in $11 / 15$ studies. The effect of progesterone-only contraceptives were less clear; of 21 studies, 8 showed a positive effect, 8 a negative effect and 5 a neutral effect. In particular, the effect of Depo-Provera was negative in 7 , positive in 4 , and neutral in 2 studies. All 5 studies investigating HRT-use demonstrated a positive influence of HRT-use on the VM.

Conclusion Exogenous sex steroids, particularly containing oestrogen, may play a role in supporting an optimal VM in both reproductive-aged and peri/post-menopausal women.

\section{P081 SIGNIFICANT DIFFERENCE IN MACROLIDE AND FLUOROQUINOLONE RESISTANCE IN MYCOPLASMA GENITALIUM IN METROPOLITAN AND OVERSEAS FRANCE IN 2018 AND 2019}

${ }^{1,2}$ S Pereyre ${ }^{*},{ }^{1} \mathrm{C}$ Laurier-Nadalié, ${ }^{2} \mathrm{C}$ Le Roy, ${ }^{2} \mathrm{~N}$ Hénin, ${ }^{1} \mathrm{M}$ Gardette, ${ }^{1,2} \mathrm{C}$ Bébéar. ${ }^{1}$ National Reference Center for bacterial STI, Bordeaux university hospital, Bordeaux, France; ${ }^{2}$ University of Bordeaux, Bordeaux, France

10.1136/sextrans-2021-sti.213

Background Limited macrolide and fluoroquinolone resistance data are available in France for Mycoplasma genitalium. The aim of this study was to investigate the prevalence of macrolide and fluoroquinolone resistance in $\mathrm{M}$. genitalium-positive men and women seeking care in metropolitan and overseas France in 2018 and 2019.

Methods A one-month systematic prospective collection of $\mathrm{M}$. genitalium-positive specimens was proposed between September 15th and October 15th 2018 and 2019 to metropolitan French diagnostic laboratories. A similar three-month collection between August 1st and October 31th 2018 and 2019 was proposed to overseas French microbiology diagnostic laboratories (La Réunion, Mayotte, French Guiana, French Polynesia, and New Caledonia). Macrolide resistance-associated mutations were detected using the ResistancePlus MG assay (SpeeDx) and 23S rRNA sequencing. Fluoroquinolone resistance-associated mutations in the parC gene were searched by sequencing.

Results A total of 1361 specimens from 1328 patients were analyzed. In metropolitan France, macrolide resistance was $42.9 \%$ and $34.7 \%$ in 2018 and 2019, respectively, and was significantly higher in men $(59.6 \%$ and $52.4 \%)$ than in women $(18.7 \%$ and $15.9 \%$, respectively, $\mathrm{p}<0.001)$. These percentages of macrolide resistance were significantly higher than those of $6.1 \%$ and $14.7 \%$ observed in overseas France in 2018 and 2019, respectively, $(\mathrm{p}<0.001)$, with no significant difference between men and women.

Regarding fluoroquinolone resistance, rates of resistance rates were significantly higher in metropolitan France in 2018 and 2019 (16.1\% and 14.9\%, respectively) than in overseas France $(1.3 \%$ and $2.6 \%$ in 2018 and 2019, respectively, $\mathrm{p}<0.001)$. No difference was observed between men and women.

Conclusion Macrolide and fluoroquinolone resistance rates are high in metropolitan France and contrast with significant lower rates in overseas France. In metropolitan France, macrolide resistance is up to $60 \%$ in men but three times lower in women, highlighting that gender and sexual behavior should be taken into account for the management of M. genitalium infections.

\section{P082 ASSESSING THE IMPACT OF THE CORONAVIRUS-19 (COVID-19) PANDEMIC ON INTERNET SEARCHES FOR SEXUALLY TRANSMITTED INFECTIONS IN THE UNITED STATES, 2019-2020}

L Haderxhanaj*, K Renfro, M Hogben, S Aral. Centers for Disease Control and Prevention, Atlanta, USA

\subsection{6/sextrans-2021-sti.214}

Background Novel approaches to monitoring sexually transmitted infections (STI) may contribute to understanding of STI rates and associated behaviors, especially during the coronavirus (COVID)-19 pandemic, when healthcare and surveillance systems are likely affected. Therefore, we explored public interest in STI-related internet search terms from 2019-2020.

Methods We downloaded Google Trends data for the United States (US) to capture number of searches including at least one of 64 search terms within four STI-related categories (STIs, symptoms, testing, and treatment) from January 1, 2019 to December 31, 2020. We expressed search interest across weeks as the unit of analysis, with scores normalized from $0-100 ; 100$ represents the week with the most search interest for each variable.

Results For many STI-related search terms, interest dropped in March 2020 (when most states deployed COVID mitigation measures), increased later in the year, but did not increase to pre-pandemic interest. For example, 'chlamydia' ranged from 73-100 from January 2019-February 2020, dropped to a low of 55 in mid-April before increasing to values between 59-79 through December. Only a few search terms did not change during the pandemic. For example, besides one peak week of 100, 'syphilis' ranged from 21-35. Although the COVID-19 pandemic shifted interest away from STIs for spring and summer of 2020, most search terms remained used throughout July to December 2020 (ranges for chlamydia: 60-79, gonorrhea: 32-44, herpes: 44-55, HPV: 38-57, STD: 60-92, and STI: 58-80).

Conclusions In the US, internet searches for STI-related terms prior to and during the COVID-19 pandemic dropped from March through fall 2020. Concerns over STI symptoms, testing, and treatment increased by fall and winter 2020, which may indicate a need for access to STI services. Google Trends is a novel way to understand public interest in health topics, especially during a global pandemic.

\section{P083 TREATMENT OF MYCOPLASMA GENITALIUM INFECTION ACCORDING TO QPCR DETERMINATION OF RESISTANCE MUTATIONS: PRACTICE-BASED CASES}

${ }^{1,2} \mathrm{~N}$ Bolshenko, 'E Goloveshkina, 'E Shedko*. 'Central Research Institute of Epidemiology, Moscow, Russian Federation; ${ }^{2}$ Moscow Regional Clinical Skin and Venereal Diseases Dispensary, Moscow, Russian Federation

\subsection{6/sextrans-2021-sti.215}

Purpose Mycoplasma genitalium 23S rRNA and parC genes are associated with antimicrobial resistance. In our study successful treatment of $\mathrm{M}$. genitalium infections chosen according 
to additional tests on resistance mutations with AmpliSens ${ }^{\circledR}$ M.genitalium-ML/FQ-Resist-FL (Central Research Institute of Epidemiology, Russia) is shown.

Approach We included 13 clinical cases of M. genitalium infection with prevalence of men practicing sex with men patients. Clinical samples were collected from urethra, oropharynx and rectum. Extraction was performed using DNAsorb-AM (AmpliSens, Russia). Identification of M. genitalium was performed using «AmpliSens ${ }^{\circledR}$ N.gonorrhoeae/C.trachomatis/M.genitalium/T.vaginalis-MULTIPRIME-FRT» (CRIE, Russia). Resistance-associated mutations were detected using AmpliSens $^{\circledR}$ M.genitalium-ML/FQ-Resist-FL (CRIE, Russia) with validation against Sanger sequencing.

Impact M. genitalium was detected in all 13 studied cases of which $46.1 \%(n=6)$ in urethral swabs, 38.5\% $(n=5)$ in rectal swabs, and two with multiple sites of infection, including oropharynx. Most patients did not show clinical signs of M. genitalium infection while others reported urethral itching (7.7\%), anal region pain (7.7\%), and rectum bloody discharge after defecation (7.7\%). Resistance-associated mutations were detected in ten $(76.9 \%)$ cases, while seven of them $(70 \%)$ contained both $23 \mathrm{~S}$ rRNA and parC mutations. Administrated treatment was chosen according to qPCR resistance mutations test and consisted of Doxycycline or Minocycline $(100 \mathrm{mg}$ orally 2 times a day for 10 days) for resistant M. genitalium, and Josamycin (500 mg orally 3 times a day for 10 day) for non-resistant $M$. genitalium. In all cases infection was completely eradicated after treatment.

Innovation and Significance Determination of M. genitalium resistance associated mutations recommended both in European guideline on Mycoplasma genitalium infections and in BASHH UK national guideline. With high prevalence of both macrolides and fluoroquinolones associated mutations in our study authors imply that application of AmpliSens ${ }^{\circledR}$ M.genitalium-ML/FQ-Resist-FL kit with simultaneous detection of both 23 rRNA and parC genes mutations is a helpful tool to a clinical practice.

\section{P084 SCREENING OF ANORECTAL AND OROPHARYNGEAL SAMPLES FAILS TO DETECT BACTERIOPHAGES INFECTING NEISSERIA GONORRHOEAE}

1J Laumen*, 'S Abdellati, 'SS Manoharan-Basil, ${ }^{1,2} \mathrm{C}$ Van Dijck, ${ }^{1} \mathrm{D}$ Van den Bossche, ${ }^{1} \mathrm{D}$ Baetselier, ${ }^{1} \mathrm{~T}$ De Block, ${ }^{2} \mathrm{~S}$ Malhotra-Kumar, ${ }^{1,3} \mathrm{P}$ Soentjens, ${ }^{4} \mathrm{~J}$ Pirnay, ${ }^{1,5} \mathrm{C}$ Kenyon, ${ }^{4,6} \mathrm{M}$ Merabishvili. ${ }^{1}$ Institute of Tropical Medicine, Department of Clinical Sciences, Antwerp, Belgium; 'Laboratory of Medical Microbiology, Vaccine and Infectious disease Institute, University of Antwerp, Antwerp, Belgium; ${ }^{3}$ Center for Infectious diseases, Queen Astrid Military Hospital, Neder-over-Heembeek, Brussels, Belgium; ${ }^{4}$ Laboratory for Molecular and Cellular Technology (LabMCT), Queen Astrid Military Hospital, Neder-over-Heembeek, Brussels, Belgium; ${ }^{5}$ Department of Medicine, University of Cape Town, Cape Town, South Africa; ${ }^{6}$ Microbiology and Virology (EIBMV), Eliava Institute of Bacteriophage, Tbilisi, Georgia

10.1136/sextrans-2021-sti.216

Background There are real concerns that Neisseria gonorrhoeae may become untreatable in the near future due to the rapid emergence of antimicrobial resistance. Alternative therapies are thus urgently required. Bacteriophages active against N. gonorrhoeae could play an important role as an antibioticsparing therapy which may even reduce the selection pressure for the emergence of resistance against conventional antimicrobials. Furthermore, they could be used in combination with antimicrobial agents to treat multi-resistant N. gonorrhoeae.
To the best of our knowledge, no phages active against $\mathrm{N}$. gonorrhoeae have ever been found.

Methods The aim of this study was to screen for bacteriophages able to lyze N. gonorrhoeae in 194 oropharyngeal and 18 anorectal ESwabsTM of 74 men who have sex with men attending a sexual health clinic in Antwerp, Belgium. ESwabsTM were enriched for bacteriophages using 11 different clinical N. gonorrhoeae strains. The spot test method was used as an initial indicator test to screen for the presence of phages by measuring lytic activity.

Results Multiple clear zones were detected as a result of antibacterial activity, but none of the confluent lysis zones could be replicated through further propagation. We screened 212 swabs but were unable to identify an anti-gonococcal bacteriophage.

Conclusion This is the first report of a large-scale screening that systematically searched for anti-gonococcal phages directly from clinical swabs. A strictly virulent $\mathrm{N}$. gonorrhoeae phage would be of considerable utility in the fight against multi resistant N. gonorrhoeae infections. Further studies may derive more success by screening for phages at other anatomical sites (e.g., stool samples, urine) or in environmental settings (e.g., toilet sewage water of sex clubs or sexually transmitted infection clinics).

\section{P087 SPATIAL AND TEMPORAL EPIDEMIOLOGY OF INFECTIOUS SYPHILIS IN VICTORIA, AUSTRALIA, 2015- 2018}

${ }^{1}$ E Aung ${ }^{*},{ }^{1,2} \mathrm{M}$ Chen, ${ }^{1,2} \mathrm{C}$ Fairley, ${ }^{3} \mathrm{~N}$ Higgins, ${ }^{4} \mathrm{D}$ Williamson, ${ }^{5} \mathrm{~J}$ Tomnay, ${ }^{1} \mathrm{~K}$ Cook, ${ }^{1} \mathrm{~J}$ Peel, ${ }^{1} \mathrm{~V}$ Dharmakulasinghe, ${ }^{3} \mathrm{C}$ Alpren, ${ }^{1,2,6} \mathrm{E}$ Chow. ${ }^{1}$ Melbourne Sexual Health Centre, Melbourne, Australia; ${ }^{2}$ Central Clinical School, Faculty of Medicine, Nursing and Health Sciences, Monash University, Melbourne, Australia; ${ }^{3}$ Department of Health and Human Services, Melbourne, Australia; ${ }^{4}$ Microbiological Diagnostic Unit Public Health Laboratory, Department of Microbiology and Immunology, The University of Melbourne at The Doherty Institute for Infection and Immunity, Melbourne, Australia; ${ }^{5}$ Centre for Excellence in Rural Sexual Health, Melbourne Medical School, The University of Melbourne, Melbourne, Australia; ${ }^{6}$ Centre for Epidemiology and Biostatistics, Melbourne School of Population and Global Health, The University of Melbourne, Melbourne, Australia

10.1136/sextrans-2021-sti.217

Background The study aims to examine the trends of syphilis infection in Victoria and the characteristics of notified cases of syphilis among different population groups stratified by risk and in different geographical distributions.

Methods We analysed the demographic characteristics, notification trends and geographical distribution of notified cases of syphilis in Victoria between 2015 and 2018.

Infectious syphilis cases were categorised into four population groups: men who have sex with men (MSM), men who have sex with women (heterosexual males), females, and men who have sex with men and women (bisexual males). We examined the staging of syphilis, geographic location by residence of cases, HIV status, reasons for testing, and notifying source.

Results Of the 4,808 notified infectious syphilis cases, there were 3,801 (64\%) MSM, 593 (12\%) heterosexual males, 465 (10\%) females, and $118(2 \%)$ bisexual males. Females $(219 \%$ increase, ptrend $<0.001)$ and bisexual males $(220 \%$ increase, ptrend $=0.004$ ) had the greatest increase in the number of cases, followed by heterosexual males $(129 \%$ increase, ptrend $<0.001)$ and MSM $(21 \%$ increase, ptrend $<0.001)$. Geographical mapping showed the majority of the syphilis cases in 